\title{
Effects of basal stem rot on oil palm inter-frond angles for different severity levels
}

\begin{abstract}
Basal Stem Rot (BSR) is the most destructive disease of oil palm in Malaysia. White-rot fungus Ganoderma is the causal pathogen of BSR disease leading to significant economic loss to the oil palm industry. Early disease detection is crucial to control the disease and to reduce the disease effects on the yield. Terrestrial Laser Scanning (TLS) is an active imaging method which is contact-free, precise, cost-effective and user-friendly. It provides accurate information on tree dimensions and morphology, which relates to plant development and health. This study proposed an image processing technique using the point clouds, ground input data taken from the terrestrial laser scanner that has capability to get a top view image of the tree. The objective of this study was to analyze the oil palm inter-frond angles (measured in degrees) using top view image of oil palm tree at four different severity levels of infection. From the results, mean degree of frond angle in each level showed significant relationship with severity level of BSR with high correlation, R2 values of 0.96. Results from Analysis of Variance (ANOVA) for frond angles exhibited significant differences between all levels of infection with p-value less than 0.0001 .
\end{abstract}

Keyword: Lidar; Point cloud; Image processing; Frond angle; Terrestrial laser scanner 